Annals of Pure and Applied Mathematics

Vol. 17, No. 1, 2018, 1-13

ISSN: 2279-087X (P), 2279-0888(online)

Published on 3 April 2018

Annals of

www.researchmathsci.org

DOI: http://dx.doi.org/10.22457/apam.v17n1al

Pure and Applied

Mathematics

\title{
An Analysis on the Causes of Breast Cancer in Women Using Fuzzy Soft Covering Based Rough Sets and its Applications
}

\author{
Anjan Mukherjee and Somen Debnath \\ Department of Mathematics, Tripura University \\ Suryamaninagar, Agartala-799022, Tripura, INDIA \\ email: anjan2002_m@yahoo.co.in, somen008@rediffmail.com \\ Received 1 March 2018; accepted 31 March 2018
}

\begin{abstract}
Breast cancer normally occurs in women and most of it begin in the ducts that carry milk to the nipple. It is the second leading cause of cancer death in women. It causes abnormal cell growth in the breast and to spread other parts of the body. These cells form a tumor called malignant (cancerous). There are many risk factors which can increase the chance of developing breast cancer. Being a woman, getting older and genetic changes are the major risk factors for breast cancer. But it is not yet known exactly how some of these risk factors cause cells to cancerous. Imaging tests such as mammograms, ultrasound, MRI etc used to detect the breast disease. The modern researchers and technological advancements attempted to determine the cause and prevention of breast cancer in an effective manner with least number of attributes. Biopsy is the only sure way to diagnosis breast cancer. But the diagnosis is lengthy process with multiple and multilevel attribute analysis in certain cases. In order to improve the accuracy of diagnosis with limited attributes, in this paper fuzzy soft covering based rough sets are used and form an algorithm to reduce the number of attributes using equivalence relation.

In this article, we investigate a data of 50 patients from different sources to find the patient/s with high breast cancer risk by using fuzzy soft covering based rough sets with the help of experts.
\end{abstract}

Keywords: Fuzzy set; Soft set; Rough set; fuzzy soft covering based rough sets; medical diagnosis.

AMS Mathematics Subject Classification (2010): 03E28, $03 \mathrm{E} 72$

\section{Introduction}

The term 'vague' is common in recent times as the problems in real life situation becomes complicated day by day. It is used in different areas such as medical application, engineering, economics pharmacology etc. Fuzzy sets, soft sets, rough sets etc are the mathematical tools to solve the problems based on vagueness. This is because of the incapability of classical mathematics to handle those problems. Many researchers are working hard and developing new theories and propose model to reshape the problems so 


\section{Anjan Mukherjee and Somen Debnath}

that it is easy to understand. In every time our aim is how to get more accurate and reliable result by using modern mathematical tools.

The most successful theoretical approach to vagueness is fuzzy set theory introduced by Zadeh in 1965[12]. It is based on the fuzzy membership function. But it is not possible to set membership function in each particular case. For the need of the situation it has been extended to intuitionistic fuzzy set [1], interval valued fuzzy set [7] and interval valued intuitionistic fuzzy set [2] etc.

In order to takel these types of situation Molodtsov in 1999, introduced soft set theory [10], Maji et al. [8] defined a hybrid model called fuzzy soft sets. This new model is a combination of fuzzy and soft sets and is a generalization of soft sets. Irfan Ali et al. [3] developed the theory.

In 1982, Pawlak [11] introduced the concept of rough set as a formal tool for modeling and processing incomplete information in information systems. There are two basic elements in rough set theory, crisp set and equivalence relation, which constitute the mathematical basis of rough sets. When handling continuous data with Pawlak model, the cost of computation becomes very high. Hence to overcome this problem several extensions of rough set theory were introduced for better approximation.

In 2010, Feng et al. [4,5,6] investigated the concept of soft rough set which is a combination of soft set and rough set.

In this paper, we study the concept of fuzzy soft covering based rough set which is a combination of covering fuzzy soft set and rough set. Here we establish a fuzzy soft covering approximation space. We put up an example to show that the new type of fuzzy soft rough set which is based on covering fuzzy soft set is more accurate than fuzzy soft rough set.

We give a new method which determine the necessity of biopsy and it gives to user a range of the risk of the cancer. For this process, it is used as laboratory data, cancer antigen 15-3(CA 15-3), scarcino embrayonic antigen (CEA), tissue polypeptide specific antigen (TPS), breast volume $(B V)$ and age of the patient. We observe that this method is more rapid, economical and without risk than the traditional diagnostic methods.

\section{Preliminaries}

In this section, we discuss basic concepts which are useful and significant in the later part of this article.

Definition 2.1. [12] Let $U$ be a universe set . A fuzzy set $A$ in $U$ is a set of ordered pairs: $A=\left\{\left(x, \mu_{A}(x)\right): x \in U\right\}$, where $\mu_{A}: U \rightarrow[0,1]=I$ is a mapping and $\mu_{A}(x)$ states the degree of belongness of $x$ in $A$. The family of all fuzzy sets in $\mathrm{U}$ is denoted by $I^{U}$.

Definition 2.2. [10] Let $U$ be an initial universe and $E$ be a set of parameters. Let $P(U)$ denotes the power set of $U$ and $A \subseteq E$. Then the pair $(F, A)$ is called soft set over $U$, where $F$ is a mapping given by $F: A \rightarrow P(U)$. 
An Analysis on the Causes of Breast Cancer in Women Using Fuzzy Soft Covering Based Rough Sets and its Applications

Definition 2.3. [11] Let $U$ be finite set and $R$ an equivalence relation on $U$. Then the pair $(U, R)$ is called a Pawlak approximation space.

$R$ generates a partition $U / R=\left\{Y_{1}, Y_{2}, \ldots \ldots, Y_{m}\right\}$ on $U$, where $Y_{1}, Y_{2}, \ldots \ldots, Y_{m}$ are the equivalence classes generated by the equivalence relation $R$. In the rough set theory, these are also called elementary sets of $R$.

For any $X \subseteq U$, we can describe $X$ by the elementary sets of $R$ and two sets

$\underline{R}(\mathrm{X})=\cup\left\{Y_{i} \in U / R: Y_{i} \subseteq X\right\}, \bar{R}(\mathrm{X})=\cup\left\{Y_{i} \in U / R: Y_{i} \cap X \neq \varnothing\right\}$

which are called the lower and upper approximation of $X$, respectively.

In addition,

$$
\begin{aligned}
& \operatorname{Pos}(X)=\underline{R}(X), \operatorname{Neg}(X)=U-\bar{R}(X) \\
& \operatorname{Bnd}(X)=\bar{R}(X)-\underline{R}(X)
\end{aligned}
$$

are called the positive, negative and boundary regions of $X$, respectively.

Definition 2.4. [11] If $\operatorname{Bnd}(X) \neq \varnothing$, that is $\bar{R}(X)=\underline{R}(X)$,

$X$ is said to be rough (or inexact); in the opposite case, that is, if the boundary region of $X$ is empty, that is, $\bar{R}(X)=\underline{R}(X)$, then $X$ is called definable (or crisp).

Definition 2.5. [9] Let $A \subseteq E$. $\left(f_{A}, E\right)$ is defined to be a fuzzy soft set on $(U, E)$ if $f_{A}: E \rightarrow I^{U}$ is mapping defined by $f_{A}(e)=\mu_{f_{A}}^{e}$, where $\mu_{f_{A}}^{e}=\bar{o}$ if $e \in E-A$ and $\mu_{f_{A}}^{e} \neq \bar{o}$ if $e \in A$, where $\bar{O}(u)=0$ for each $u \in U$.

Example 2.6. Virat Kohli and Anushka Sharma went to Italy to marry and for this they want to hire a wedding room. The fuzzy soft set $\left(f_{A}, E\right)$ describes the "capacity of the wedding room". Let $U=\{x, y, z, m, n\}$ be the wedding rooms under consideration. Let $E=\{$ $\left.e_{1}, e_{2}, e_{3}, e_{4}, e_{5}\right\}$ be the set of parameters which stand for big, expensive, elegant, quality and good serving respectively and $A=\left\{e_{1}, e_{2}, e_{5}\right\}$ a subset of $E$. Then the fuzzy soft set on $(U, E)$ is denoted by $\left(f_{A}, E\right)$ and it is defined as $\left(f_{A}, E\right)=\left\{e_{1}=\{x / 0.5, y / 0.8, z / 0.6, m / 0.8, n / 0.4\}, e_{2}=\{x / 0.3, y / 0.5, z / 0.6, m / 0.8, n / 0.9\}, e_{5}\right.$ $=\{x / 0.8, y / 0.6, z / 0.3, m / 0.4, n / 0.5\}\}$.

\section{Fuzzy soft covering based rough set}

Here we use a different kind of fuzzy soft set with rough set and establish a fuzzy soft covering approximation space and present its basic properties. 


\section{Anjan Mukherjee and Somen Debnath}

Definition 3.1. Let $G=\left(f_{A}, E\right)$ where $A \subseteq E$ be a fuzzy soft set over $U$. Then the pair $S=$ $(U, G)$ is called a fuzzy soft approximation space.

Based on $S$ we define the following two operations:

$$
\begin{aligned}
& f_{\text {aprs }}(X)=\left\{u \in U: \exists a \in A, u \in f_{A}(a) \subseteq X\right\} \\
& f_{\overline{a p r^{S}}}(X)=\left\{u \in U: \exists a \in A, u \in f_{A}(a), f_{A}(a) \cap X \neq \varnothing\right\}
\end{aligned}
$$

which are called the fuzzy soft $S$-lower approximation and the fuzzy soft $S$-upper approximation of $X$ where $X$ is a fuzzy soft set over $U$.

In general, we consider $f_{a p r_{S}}(X)$ and $f_{\overline{a p r^{S}}}(X)$ as fuzzy soft rough approximations of $X$ with respect to $S$.

So in addition we can write

$$
\begin{aligned}
& \operatorname{Pos}_{S}(X)=\underline{f_{a p r_{S}}}(X) \\
& \operatorname{Neg}_{S}(X)=U--f_{\overline{a p r^{S}}}(X) \\
& \operatorname{Bnd}_{S}(X)=f \overline{a p r^{S}}(X)-f_{a p r_{S}}(X)
\end{aligned}
$$

Are called the fuzzy soft $S$-positive, negative and boundary regions of $X$ respectively. If $f_{\overline{a p r^{s}}}(X)=f_{a p r_{S}}(X), X$ is said to be fuzzy soft $S$-definable otherwise $X$ is called fuzzy soft $S$-rough set.

Definition 3.2. Let $G=\left(f_{A}, E\right)$ be a fuzzy soft set over $U$ and $S=(U, G)$ a fuzzy soft approximation space. Then we have

$$
\begin{aligned}
& \frac{f_{a p r_{S}}}{}(X)=\underset{e \in A}{\cup}\left\{f_{A}(e): f_{A}(e) \subseteq X\right\} \\
& f_{\overline{a p r^{s}}}(X)=\cup\left\{f_{e \in A}(e): f_{A}(e) \cap X \neq \varnothing\right\}
\end{aligned}
$$

We have the following properties related to the above approximations.

\section{Results 3.3.}

1) $\underline{f_{\text {aprs }}}(\varnothing)=f_{\frac{a_{\text {apr }}^{s}}{}}(\varnothing)=\varnothing$

2) $\underline{f_{a p r_{S}}}(U)=f_{\frac{a p r^{s}}{}}(U)=\cup_{e \in A} f_{A}(e)$

3) $f_{\text {apr }}(X) \subseteq X$

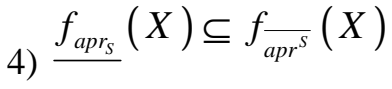

5) $X \subseteq Y \Rightarrow \underline{f_{a p r_{s}}}(X) \subseteq \underline{f_{a p r_{s}}}(Y)$

6) $X \subseteq Y \Rightarrow f_{\overline{a p r^{s}}}(X) \subseteq f_{\overline{a p r^{s}}}(Y)$

7) $\underline{f_{a p r_{S}}}(X \cap Y) \subseteq \underline{f_{a p r_{S}}}(X) \cap \underline{f_{a p r_{S}}}(Y)$

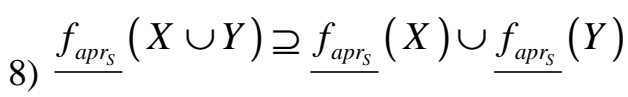


An Analysis on the Causes of Breast Cancer in Women Using Fuzzy Soft Covering Based Rough Sets and its Applications

9) $f_{\overline{a p r^{s}}}(X \cup Y)=f_{\overline{a p r^{s}}}(X) \cup f_{\overline{a p r^{s}}}(Y)$

10) $f_{\overline{a p r^{s}}}(X \cap Y) \subseteq f_{\overline{a p r^{s}}}(X) \cap f_{\overline{a p r^{s}}}(Y)$

Definition 3.4. A full fuzzy soft set $G=\left(f_{A}, E\right)$ where $A \subseteq E$ over $(U, E)$ is called a covering fuzzy soft set if $f_{A}(a) \neq \varnothing, \forall a \in A$. We denote a covering fuzzy soft set as $C F_{G}$.

Definition 3.5. Let $G=\left(f_{A}, E\right)$ be a covering fuzzy soft set over $U$. Then the pair $\left(U, C F_{G}\right)$ is called a fuzzy soft covering approximation space.

Definition 3.6. Let $S=\left(U, C F_{G}\right)$ be a fuzzy soft covering approximation space and $x \in U$. Then the fuzzy soft minimal description of $x$ is defined as $\operatorname{Md}_{F S}(x)=\left\{f_{A}(a): a \in A \wedge x \in f_{A}(a) \wedge\left(\forall e \in A \wedge x \in f_{A}(e) \subseteq f_{A}(a) \Rightarrow f_{A}(a)=f_{A}(e)\right)\right\}$

Definition 3.7. Let $S=\left(U, C F_{G}\right)$ be a fuzzy soft covering approximation space. For a set $X \subseteq U$, fuzzy soft covering lower and upper approximations are, respectively, defined as

$$
\begin{gathered}
F S_{*}(X)=\cup_{e \in E}\left\{f_{A}(e): f_{A}(e) \subseteq X\right\} \\
\overline{F S^{*}}(X)=\cup\left\{M d_{F S}(x): x \in X\right\} .
\end{gathered}
$$

Definition 3.8. Let $S=\left(U, C F_{G}\right)$ be a fuzzy soft covering approximation space. A subset $X \subseteq U$ is called fuzzy soft covering based definable if $\underline{F S_{*}}(X)=\overline{F S^{*}}(X)$, otherwise it is called fuzzy soft covering based rough set.

Example 3.9. Let $U=\left\{u_{1}, u_{2}, u_{3}, u_{4}, u_{5}, u_{6}\right\}$ be a universal set and $G=\left(f_{A}, E\right)$ a covering fuzzy soft set over $U$, where $E=\left\{e_{1}, e_{2}, e_{3}, e_{4}, e_{5}, e_{6}\right\}$ such that

$$
\begin{aligned}
& f_{A}\left(e_{1}\right)=\left\{u_{1} / 0.5, u_{2} / 0.4\right\} \\
& f_{A}\left(e_{2}\right)=\left\{u_{1} / 0.3, u_{2} / 0.5, u_{3} / 0.6, u_{4} / 0.8, u_{5} / 0.8, u_{6} / 0.4\right\} \\
& f_{A}\left(e_{3}\right)=\left\{u_{3} / 0.2, u_{4} / 0.6\right\} \\
& f_{A}\left(e_{4}\right)=\left\{u_{3} / 0.6, u_{4} / 0.8, u_{5} / 0.3, u_{6} / 0.7\right\} \\
& f_{A}\left(e_{5}\right)=\left\{u_{1} / 0.4, u_{2} / 0.3, u_{5} / 0.6, u_{6} / 0.3\right\} \\
& f_{A}\left(e_{6}\right)=\left\{u_{3} / 0.3, u_{5} / 0.7, u_{6} / 0.5\right\}
\end{aligned}
$$

Then, $S=\left(U, C F_{G}\right)$ is a fuzzy soft covering approximation space.

For $X_{1}=\left\{u_{1} / 0.5, u_{2} / 0.4\right\}$ we have 


\section{Anjan Mukherjee and Somen Debnath}

$F S_{*}\left(X_{1}\right)=\left\{u_{1} / 0.5, u_{2} / 0.4\right\}$

$\overline{F S^{*}}\left(X_{1}\right)=\left\{u_{1} / 0.5, u_{2} / 0.4\right\}$

Thus FS $\left(X_{1}\right)=\overline{F S^{*}}\left(X_{1}\right)$ and $X_{1}$ is a fuzzy soft covering based definable set.

Again, for $X_{2}=\left\{u_{1} / 0.5, u_{2} / 0.4, u_{4} / 0.6\right\}$

$F S_{*}\left(X_{2}\right)=\left\{u_{1} / 0.5, u_{2} / 0.4\right\}$

$\overline{F S^{*}}\left(x_{2}\right)=\left\{u_{1} / 0.5, u_{2} / 0.4, u_{3} / 0.6, u_{4} / 0.8, u_{5} / 0.3, u_{6} / 0.7\right\}$

Thus $F S_{*}\left(X_{2}\right) \neq \overline{F S^{*}}\left(X_{2}\right)$ and $X_{2}$ is a fuzzy soft covering based rough set.

A fuzzy soft rough set is based on a fuzzy soft set in a fuzzy soft approximation space, whereas a fuzzy soft covering based rough set is based on a covering fuzzy soft set in a fuzzy soft covering approximation space.

Corollary 3.10. Let $G=\left(f_{A}, E\right)$ be a covering fuzzy soft set over $U$ and $S=\left(U, C F_{G}\right)$ a fuzzy soft covering approximation space and $X, Y \subseteq U$. Then the fuzzy soft covering lower and upper approximations do not have the following properties:

1) $F S_{*}(X \cap Y)=F S_{*}(X) \cap F S_{*}(Y)$

2) $F S(\sim F S(X))=\sim F S(X)$

$-\overline{F S^{*}}\left(\sim-\overline{F S^{*}}(X)\right)=\sim \overline{F S^{*}}(X)$

4)

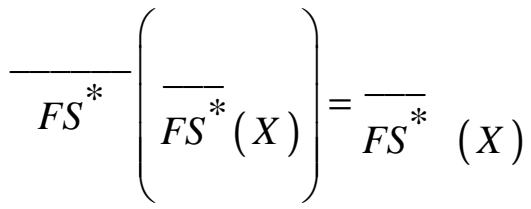

5) $F S \quad(X)=\sim \overline{F S^{*}}(\sim X)$

6) $\overline{F S^{*}}(X)=\sim F S \quad(\sim X)$

7) $\forall a \in A, \overline{F S^{*}}\left(f_{A}(a)\right)=f_{A}(a)$

The following examples give the justification of the corollary 3.10 
An Analysis on the Causes of Breast Cancer in Women Using Fuzzy Soft Covering Based Rough Sets and its Applications

Let $U=\left\{u_{1}, u_{2}, u_{3}, u_{4}, u_{5}\right\}$ be universe $G=\left(f_{A}, E\right)$ a covering fuzzy soft set over $U$ and $E=\left\{e_{1}, e_{2}, e_{3}, e_{4}\right\}$ be a set of parameter such that

$f_{A}\left(e_{1}\right)=\left\{u_{1} / 0.4, u_{2} / 0.6\right\}$

$f_{A}\left(e_{2}\right)=\left\{u_{2} / 0.3, u_{3} / 0.5\right\}$

$f_{A}\left(e_{3}\right)=\left\{u_{3} / 0.3, u_{4} / 0.2\right\}$

$f_{A}\left(e_{4}\right)=\left\{u_{1} / 0.5, u_{2} / 0.8, u_{4} / 0.2, u_{5} / 0.7\right\}$

Then $S=\left(U, C F_{G}\right)$ is a fuzzy soft covering approximation space.

1) Assume that $X=\left\{u_{1} / 0.5, u_{2} / 0.8\right\}$ and $Y=\left\{u_{2} / 0.4, u_{3} / 0.5, u_{4} / 0.7\right\} \subset U$

$F S_{*}(X)=\left\{u_{1} / 0.4, u_{2} / 0.6\right\}$,

and $F S_{*}(Y)=\left\{u_{2} / 0.3, u_{3} / 0.5, F S_{*}(X) \cap F S_{*}(Y)=\left\{u_{2} / 0.3\right\} . F S_{*}(X \cap Y)=\phi\right.$.

Therefore, $F S \quad(X \cap Y) \neq \overline{F S} \quad \overline{(X)} \cap \quad F S \quad(Y)$

2) Suppose $X=\left\{u_{1} / 0.3, u_{2} / 0.5, u_{3} / 0.8, u_{5} / 0.7\right\}$

$$
\text { FS } \quad(X)=\left\{u_{2} / 0.3, u_{3} / 0.5\right\}
$$

$\sim F S(X)=\left\{u_{1} / 0.3, u_{5} / 0.7\right\}$

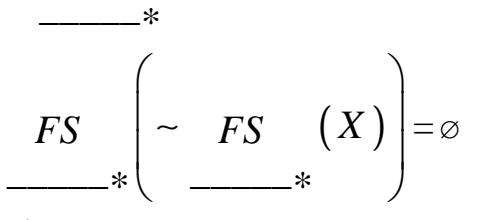

Thus,

$$
\underset{F S}{-}(\sim F S(X)) \neq \sim \text { FS }(X)
$$

3) Let $X=\left\{u_{1} / 0.4, u_{2} / 0.5\right\}$

$$
\begin{aligned}
& \overline{F S^{*}}(X)=\left\{u_{1} / 0.5, u_{2} / 0.8, u_{4} / 0.2, u_{5} / 0.7\right\} \\
& \overline{F S^{*}}(X)=\phi \\
& \overline{F S^{*}}\left(\sim \overline{F S^{*}}(X)\right)=\left\{u_{1} / 0.5, u_{2} / 0.8, u_{3} / 0.5, u_{4} / 0.2, u_{5} / 0.7\right\}
\end{aligned}
$$


Anjan Mukherjee and Somen Debnath

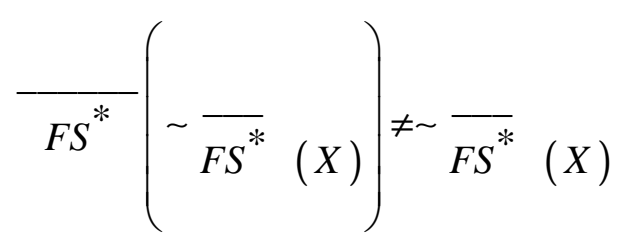

4) Suppose $X=\left\{u_{1} / 0.6, u_{2} / 0.7\right\}$

$$
\begin{aligned}
& \overline{F S^{*}}(X)=\varnothing \\
& \overline{F S^{*}}\left(\overline{F S^{*}(X)}\right)=\left\{u_{1} / 0.5, u_{2} / 0.8, u_{3} / 0.5, u_{4} / 0.2, u_{5} / 0.7\right\} \\
& \overline{F S^{*}}\left(\overline{F S^{*}}(X)\right) \neq \overline{F S^{*}}(X) \\
& \text { 5) Let } X=\left\{u_{1} / 0.5, u_{4} / 0.2\right\} \\
& \sim \mathrm{X}=\left\{u_{2} / 1.0, u_{3} / 1.0, u_{5} / 1.0\right\} \\
& \text { FS } \quad(X)=\varnothing \\
& \overline{F S^{*}}(\sim X)=\varnothing \\
& \sim \overline{F S^{*}}(\sim X)=\left\{u_{1} / 0.5, u_{4} / 0.2\right\}
\end{aligned}
$$

Therefore, $\quad F S \quad(X) \neq \sim \overline{F S^{*}}(\sim X)$

6) $X=\left\{u_{1} / 0.4, u_{2} / 0.3, u_{3} / 0.7\right\}$

$$
\begin{aligned}
& \overline{F S^{*}}(X)=\left\{u_{1} / 0.5, u_{2} / 0.8, u_{4} / 0.2, u_{5} / 0.7\right\} \\
& \sim X=\left\{u_{4} / 1.0, u_{5} / 1.0\right\} \\
& F S \quad(\sim X)=\varnothing \\
& \sim_{* S S}(\sim X)=\left\{u_{1} / 0.4, u_{2} / 0.3, u_{3} / 0.7\right\}
\end{aligned}
$$

Thus, $\overline{F S^{*}}(X) \neq \sim F S \quad(\sim X)$

7) $f_{A}\left(e_{1}\right)=\left\{u_{1} / 0.4, u_{2} / 0.6\right\}$

$\overline{F S}{ }^{*}\left(f_{A}\left(e_{1}\right)\right)=\left\{u_{1} / 0.5, u_{2} / 0.8, u_{4} / 0.2, u_{5} / 0.7\right\}$

Therefore,

$\forall a \in A, \overline{F S^{*}}\left(f_{A}(a)\right) \neq f_{A}(a)$ 
An Analysis on the Causes of Breast Cancer in Women Using Fuzzy Soft Covering Based Rough Sets and its Applications

\section{Algorithm}

We construct an algorithm for multicriteria group decision making as follows

Step 1: Input the Fuzzy soft set $G=\left(f_{A}, E\right)$.

Step 2: Formulate the evaluation fuzzy soft set $G_{1}=\left(f_{V}, T\right)$ using the primary evaluation results of the expert group $T$.

Step 3: Compute fuzzy soft rough approximations and then obtain the fuzzy soft sets $G_{1-}=\left(f_{V_{-}}, T\right)$ and $G_{1}^{-}=\left(f_{V^{-}}, T\right)$.

Step 4: Compute the corresponding functions $\mu_{G_{1}}, \mu_{G_{1-}}$ and $\mu_{G_{1}^{-}}$of the fuzzy soft sets $G_{1}=\left(f_{V}, T\right), G_{1_{-}}=\left(f_{V_{-}}, T\right)$ and $G_{1}^{-}=\left(f_{V^{-}}, T\right)$.

Step 5: Construct the fuzzy soft set $G_{F}=(\alpha, C)$ using $\mu_{G_{1}}, \mu_{G_{1-}}$ and $\mu_{G_{1}^{-}}$.

Step 6: Input the weighting vector $R$ and compute the weighted evaluation values $w\left(u_{k}\right)$ of each alternatives $u_{k} \in U$. Then rank all the alternatives according to their weighted evaluation values, One can select any of the objects with the largest weighted evaluation value as the most preferred alternative.

We use this method to help doctors in diagnosing the breast cancer risk. In our work, we use fuzzy soft covering approximations instead of fuzzy soft rough approximations in step 3. We may expect to gain much more useful information with the help of fuzzy soft covering approximations.

\section{An application of multicriteria group decision making by new type of fuzzy soft covering approximation operators}

In this work, we use fuzzy soft covering approximations and aim to obtain the optimal choice for applying biopsy to the patients with breast cancer risk by using the $C A 15-3$, $C E A, T P S, B V$ and age data of patients. Our aim is to help the doctor to determine whether the patient needs biopsy or not.

We choose a data of 50 patients from different sources with breast complain.

Step 1: Let $U=\left\{u_{k}: u_{1}=1, u_{2}=2, \ldots \ldots \ldots, u_{50}=50, k=1,2, \ldots \ldots \ldots, 50\right\}$ be the universe and $A=\{C A 15-3, C E A, T P S, B V, A g e\}$ the parameter set. Now we obtain parameterized subsets of the universe. The patients whose $C A 15-3$ in blood is 25 and higher than 25, $C E A$ is $3(\mathrm{ng} / \mathrm{ml})$ and higher than $3(\mathrm{ng} / \mathrm{ml}), T P S$ in blood is $80 \mathrm{U} / \mathrm{L}$ and higher than 80 $\mathrm{U} / \mathrm{L}$, age is 40 to 49 are chosen with expert suggestion. We generate $G=\left(f_{A}, E\right)$ where $A \subseteq E$ which is based on $C A 15-3, C E A, T P S, B V$ and age values of patients over $U$. 


\section{Anjan Mukherjee and Somen Debnath}

Since $G=\left(f_{A}, E\right)$ is a covering fuzzy soft set, $S=\left(U, C F_{G}\right)$ is the fuzzy soft covering approximation space. Consider the following

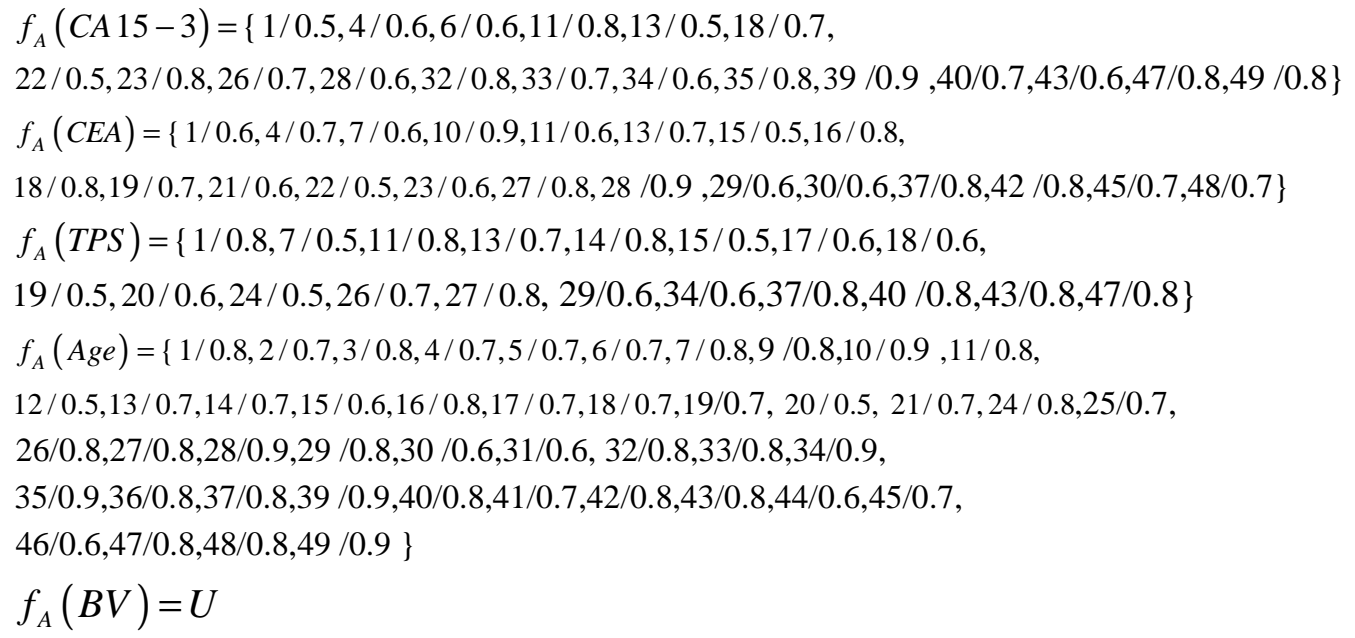

Step 2: Let $T=\left\{T_{d_{1}}, T_{d_{2}}, T_{d_{3}}\right\}$ be the specialist doctors group who evaluate the patients with respect to the parameters $C A 15-3, C E A, T P S, B V$ and age. Now we generate the fuzzy soft set $G_{1}=\left(f_{V}, T\right)$ over $U$ by using the first evaluation of the results of expert doctors group T. Each experts needs to examine all the objects in $U=\left\{u_{k}: u_{1}=1, u_{2}=2, \ldots \ldots \ldots, u_{50}=50, k=1,2, \ldots \ldots ., 50\right\}$ and will be requested to only point out "the optimal alternatives " as her evaluation result. Hence, each specialist's primary evaluation results are subsets of 50 patients from different sources with breast complain as the data. For simplicity, we assume that the evaluations of these specialist in $T=\left\{T_{d_{1}}, T_{d_{2}}, T_{d_{3}}\right\}$ are of the same importance.

$X_{d_{1}}=f_{V}\left(T_{d_{1}}\right)=\{1 / 0.5,4 / 0.7,6 / 0.6,7 / 0.7,8 / 0.6,9 / 0.8$,

11/0.9,13/0.6,15/0.5,17/0.7,18/0.7,20/0.8,22/0.6,23/0.6,25/0.7,26/0.8,

28/0.7,32/0.8,33/0.8,34/0.9,35/0.9,39 /0.9,40/0.7,41/0.7,

$43 / 0.6,47 / 0.9,49 / 0.8\}$

$X_{d_{2}}=f_{V}\left(T_{d_{2}}\right)=\{1 / 0.6,3 / 0.7,4 / 0.6,7 / 0.8,9 / 0.6,10 / 0.8,11 / 0.7$,

$12 / 0.4,13 / 0.6,14 / 0.7,16 / 0.8,18 / 0.6,20 / 0.7,22 / 0.5,24 / 0.7,25 / 0.6$,

$28 / 0.7,29 / 0.8,31 / 0.6,33 / 0.7,34 / 0.6,36 / 0.8,39 / 0.7$ $40 / 0.6,42 / 0.7,43 / 0.8,45 / 0.7,46 / 0.7,47 / 0.6,49 / 0.8\}$

$X_{d_{3}}=f_{V}\left(T_{d_{3}}\right)=\{1 / 0.5,2 / 0.7,3 / 0.8,4 / 0.6,5 / 0.7,7 / 0.6,9 / 0.8,11 / 0.6$,

13/0.7,15/0.6,16/0.8,17/0.5,18/0.7,19/0.5,20/0.6,21/0.6,22/0.5,23/0.7,

26/0.6,27/0.8,29/0.7,30/0.7,33/0.6,35/0.6,37/0.6,39/0.8, 
An Analysis on the Causes of Breast Cancer in Women Using Fuzzy Soft Covering Based Rough Sets and its Applications

40/0.6,42/0.7,43/0.8,44/0.6,47/0.7,48/0.8,49/0.7\}

Step 3: Let us choose $S=\left(U, C F_{G}\right)$ as the fuzzy soft covering approximation space. By using the fuzzy soft covering approximations, we obtain two other fuzzy soft sets $G_{1-}=\left(f_{V_{-}}, T\right)$ and $G_{1}^{-}=\left(f_{V^{-}}, T\right)$ over $U$, where $f_{V_{-}}: T \rightarrow P(U), f_{V_{-}}\left(T_{d_{i}}\right)={ }^{F S}\left(X_{d_{i}}\right), i=1,2,3$

$f_{V^{-}}: T \rightarrow P(U), f_{V^{-}}\left(T_{d_{i}}\right)=\overline{F S^{*}}\left(X_{d_{i}}\right), i=1,2,3$

$f_{V_{-}}\left(T_{d_{1}}\right)={ }^{F S}\left(X_{d_{1}}\right)=f_{A}(C A 15-3)$

$f_{V_{-}}\left(T_{d_{2}}\right)={ }^{F S}\left(X_{d_{2}}\right)=\varnothing$

$f_{V_{-}}\left(T_{d_{3}}\right)=\underline{F S}\left(X_{d_{3}}\right)=\varnothing$

$f_{V^{-}}\left(T_{d_{1}}\right)=\overline{F S^{*}}\left(X_{d_{1}}\right)=f_{A}(C A 15-3) \cup f_{A}(C E A) \cup f_{A}(T P S) \cup f_{A}($ Age $)=f_{A}($ Age $)$

$f_{V^{-}}\left(T_{d_{2}}\right)=\overline{F S^{*}}\left(X_{d_{2}}\right)=f_{A}(C A 15-3) \cup f_{A}(C E A) \cup f_{A}(T P S) \cup f_{A}($ Age $)=f_{A}($ Age $)$

$f_{V^{-}}\left(T_{d_{3}}\right)=\overline{F S^{*}}\left(X_{d_{2}}\right)=f_{A}(C A 15-3) \cup f_{A}(C E A) \cup f_{A}(T P S) \cup f_{A}($ Age $)=f_{A}($ Age $)$

Step 4: The results of the specialist three doctors evaluation can be executed in terms of fuzzy sets.

For $X \subseteq U$, the characteristic function of $X$ is denoted by $\eta_{X}$. Based on the fuzzy soft set $G_{1}=\left(f_{V}, T\right)$,we can define fuzzy set $\mu_{G_{1}}$ with the help of characteristic function $\eta$ in $U$ by

$\mu_{G_{1}}: U \rightarrow[0,1]$, 
Anjan Mukherjee and Somen Debnath

$u_{k} \rightarrow \mu_{G_{1}}\left(u_{k} / \min [0,1]\right)=\frac{1}{3} \sum_{i=1}^{3}\left[\eta_{f_{V}\left(T_{d_{i}}\right)}\left(u_{k} / \min [0,1]\right)\right]$ where $f_{V}\left(T_{d_{i}}\right)=X_{d_{i}}$ and

$\mathrm{k}=1,2, \ldots \ldots, 50$; and $i=1,2,3$.

In a similar way, we can get fuzzy sets $\mu_{G_{1-}}$ and $\mu_{G_{1}^{-}}$as follows:

$\mu_{G_{1-}}: U \rightarrow[0,1]$

$u_{k} \rightarrow \mu_{G_{1-}}\left(u_{k} / \min [0,1]\right)=\frac{1}{3} \sum_{i=1}^{3}\left[\eta_{f_{V-}\left(T_{d_{i}}\right)}\left(u_{k} / \min [0,1]\right)\right]$,

$\mu_{G_{1}^{-}}: U \rightarrow[0,1]$

$u_{k} \rightarrow \mu_{G_{1}^{-}}\left(u_{k} / \min [0,1]\right)=\frac{1}{3} \sum_{i=1}^{3}\left[\eta_{f_{v^{-}}\left(T_{d_{i}}\right)}\left(u_{k} / \min [0,1]\right)\right]$

where

$$
f_{V_{-}}\left(T_{d_{i}}\right)=\underset{F S}{F}\left(X_{d_{i}}\right), \underset{\substack{\mathrm{i}=1,2,3 \\ V^{-}}}{f_{i}}\left(T_{d_{i}}\right)=\overline{F S}^{*}\left(X_{d_{i}}\right), \text { and } \mathrm{k}=1,2, \ldots \ldots, 50
$$

From $G_{1_{-}} \subseteq G_{1} \subseteq G_{1}^{-}$, it is easy to see that $\mu_{G_{1_{-}}} \subseteq \mu_{G_{1}} \subseteq \mu_{G_{1}^{-}}$. These fuzzy sets $\mu_{G_{\mathrm{I}_{-}}} \subseteq \mu_{G_{1}} \subseteq \mu_{G_{1}^{-}}$can be interpreted by a set of parameters $C=\{L, M, H\}$ Where $L, M$ and $H$ denote some vague concepts like "the patients under low risk", "the patients under middle risk", "the patients under high risk", respectively. Now we can define a fuzzy soft set $G_{F}=(\alpha, C)$ over $U$, where $\alpha: C \rightarrow I^{U}$ is given by $\alpha(L)=\mu_{G_{\mathrm{L}_{-}}}, \alpha(M)=\mu_{G_{1}}$ and $\alpha(H)=\mu_{G_{1}^{-}}$.

For the first patient we obtain the fuzzy sets as

$\mu_{G_{1-}}(1)=\frac{1}{3}, \mu_{G_{1}}(1)=\frac{2}{3}, \mu_{G_{1}^{-}}(1)=1$

Step 5: Given a weighting vector $R=\left(R_{L}, R_{M}, R_{H}\right)$ such that $R_{L}+R_{M}+R_{H}=1$.

$w\left(u_{k}\right)=R_{L} \cdot \alpha(L)\left(u_{k}\right)+R_{M} \cdot \alpha(M)\left(u_{k}\right)+R_{H} \cdot \alpha(H)\left(u_{k}\right)$ is called the weighted evaluation value of the alternative $u_{k} \in U, k=1,2, \ldots \ldots . ., 50$. Assume the weighting vector $R=(0.3,0.4,0.3)$. Finally we can select the objects $u_{q}$ such that $w\left(u_{q}\right)=\max \left\{w\left(u_{k}\right): k=1,2, \ldots \ldots, 50\right\}$ as the patient with the highest cancer risk.

When we rank all the alternatives according to their weighted evaluation values, we can select any of the objects with the largest weighted evaluation value as the highest cancer risk. The results are as follows:

$1 \equiv 4 \equiv 15 \equiv 22 \equiv 28 \equiv 40 \equiv 42=0.67>2 \equiv 3 \equiv 5 \equiv 6 \equiv 7 \equiv 8 \equiv 9 \equiv 10 \equiv 11 \equiv 12 \equiv 13 \equiv 14 \equiv 16 \equiv$ $17 \equiv 18 \equiv 19 \equiv 20 \equiv 21 \equiv 23 \equiv 24 \equiv 25 \equiv 26 \equiv 27 \equiv 29 \equiv 30 \equiv 31 \equiv 32 \equiv 33 \equiv 34 \equiv 35 \equiv 36 \equiv 37 \equiv$ $39 \equiv 41 \equiv 43 \equiv 44 \equiv 45 \equiv 46 \equiv 47 \equiv 48 \equiv 49=0.53>38 \equiv 50=0.0$ 
An Analysis on the Causes of Breast Cancer in Women Using Fuzzy Soft Covering Based Rough Sets and its Applications

Above results show that 0.67 is the highest weightage value and 7 patients have this value and the patients with the membership 0.67 are potential cancer patients and they are under the highest risk. They need biopsy exactly. 41 patients with 0.53 value are under middle risk and they should be followed up by the doctor. The other two patientsdonot need the biopsy i.e. they are out of danger.

\section{Conclusions}

In this article, we study the concept of fuzzy soft covering based rough sets. We also define various operations on fuzzy soft covering based rough sets and prove some results on them. At the end of this paper we give an application of fuzzy soft covering based rough sets in a decision making problem where we reduce the number of patients who need biopsy.

Acknowledgements. Authors are grateful to the anonymous referees for their priceless suggestions which have improved the presentation.

\section{REFERENCES}

1. K.Atanassov, Intuitionistic fuzzy sets, Fuzzy Sets and Systems, 20 (1986) 87-96.

2. K.Atanassov and G.Gargov, Interval valued intuitionistic fuzzy sets, Fuzzy Sets and Systems, 31 (1989) 343-349.

3. M.I.Ali, F.Feng, X.Liu, W.Min and M.Shabir, On some new operations in soft set theory, Computers and Mathematics with Applications, 57 (2009) 1547-1553.

4. F.Feng, C.Li, B.Davvaz, M.I.Ali, Soft sets combined with fuzzy sets and rough setsa tentative approach, Soft Computing, 14 (2010) 899-911.

5. F.Feng, Soft rough sets applied to multicriteria group decision making, Annals of Fuzzy Mathematics and Informatics, 2 (2011) 69-80.

6. F.Feng, X.Liu, V.Leoreanu and J.B.Young, Soft sets and soft rough sets, Information Sciences, 181 (2011) 1125-1137.

7. M.Gorzalczan, A method of inference in approximate reasoning based on interval valued fuzzy sets, Fuzzy Sets and Systems, 21 (1987) 1-17.

8. P.K.Maji, R.Biswas and A.R.Roy, Soft set theory, Computers and Mathematics with Applications, 45 (2003) 555-562.

9. P.K.Maji, R.Biswas and A.R.Roy, Fuzzy soft sets, Journal of Fuzzy Mathematics, 9(2001) 589-602.

10. D.Molodtsov, Soft set theory-first results, Computers and Mathematics with Application, 37 (1999) 19-31.

11. Z.Pawlak, Rough sets, International Journal of Computer and Information Sciences, 11 (1982) 341-356.

12. L.A.Zadeh, Fuzzy set, Information and Control, 8 (1965) 338-353. 